\title{
Silvia Quendler
}

Volksschule St. Johann im Lavanttal

\section{Angelika Dobrowsky}

Praxisvolksschule Baden

\section{Lesson Study - die Rolle der Schulleitung}

DOI: https://doi.org/10.53349/sv.2021.i2.a89

Die Aufgaben der Lehrpersonen bei Lesson Study-Prozessen sind in der Literatur ausführlich dargestellt. Zusätzlich gibt es zahlreiche Veröffentlichungen von Praxisbeispielen und Erfahrungsberichten (Mewald \& Rauscher, 2019). Eine noch wenig beschriebene Rolle ist jene der Schulleitung in der praktischen Umsetzung von Lesson Study, weshalb dieser Beitrag anhand von zwei Erfahrungsberichten einen Einblick in die Aufgaben der Schulleiter*innen gibt.

\section{Lesson Study - die Rolle der Schulleitung}

Während Lesson Studies (LS) in Japan eine bereits über 100-jährige Tradition in der Lehrerfortbildung haben, erreichten sie westliche Staaten erst in den letzten Jahrzehnten. In Österreich erkannte Peter Posch das Potenzial dieses Konzepts und machte es durch Vorträge und auch durch die Übersetzung des Buches Variation Theory and the Improvement of Teaching and Learning (2012) von Mun Ling Lo bekannt. Das Handbuch von Pete Dudley (2014) zur Lesson Study wurde von Erwin-Maria Gierlinger und Thomas Wagner übersetzt. Claudia Mewald und Erwin Rauscher veröffentlichten Lesson Study: Das Handbuch für kollaborative Unterrichtsentwicklung und Lernforschung (Pädagogik für Niederösterreich 7) als erstes Handbuch in deutscher Sprache.

Lesson Study ist kollaborativ und baut auf die gemeinsame Arbeit eines Teams auf, das man auch professionelle Lerngemeinschaft (PLG) nennen kann. Die PLG hat mittlerweile in der Schul- und Unterrichtsentwicklung einen wichtigen Stellenwert. Die Zusammenarbeit als professionelle Gemeinschaft im Sinne des kollegialen Lernens ermöglicht eine kontinuierliche schulische Qualitätsentwicklung (Mewald \& Rauscher, 2019). Die PLG ist jedoch nicht iden- 
tisch mit dem Team der gesamten Schule, sie versteht sich als fach- und lerngruppenorientiert mit einem klaren Fokus auf der Verbesserung des Unterrichts (Helmke, 2012).

Die Aufgaben der Lehrpersonen bei Lesson Study-Prozessen sind in der Literatur ausführlich dargestellt. Zusätzlich gibt es zahlreiche Veröffentlichungen von Praxisbeispielen und Erfahrungsberichten (Mewald \& Rauscher, 2019). Eine noch wenig beschriebene Rolle ist jene der Schulleitung in der praktischen Umsetzung von Lesson Study, weshalb dieser Bericht einen Einblick in die Aufgaben der Schulleiter*innen gibt.

Schul- und Unterrichtsentwicklung sind Kernaufgaben der Schulleitung (vgl. Schulunterrichtsgesetz $\S 56, A b s .2)$. Auch wenn das gesamte pädagogische Team einer Schule Verantwortung für die erbrachten Lernergebnisse der Schüler*innen trägt und alle Lehrpersonen aufgefordert sind, sich in fachdidaktischer und pädagogischer Hinsicht weiterzubilden sowie konsequent an der Verbesserung des schulischen Lernangebotes mitzuarbeiten, kommt der Schulleitung eine ganz wesentliche Rolle zu. Sie ist diejenige, die Entwicklungen ankurbelt und Methoden auswählt, die für den jeweiligen Schulstandort geeignet sind. Folgende Aktivitäten fallen in das Aufgabengebiet der Schulleitung.

* Initiieren:

Auswählen und Ansprechen von Lehrpersonen, die Interesse zeigen, sodass innerhalb des Lehrer*innen-Teams einer Schule professionelle Lerngemeinschaften entstehen.

* Organisieren:

Festlegen einer Leitfrage in Hinblick auf die Verbesserung des Lernangebots.

Einladen von Wissenspartnern (externe und/oder interne Expert*innen aus unterschiedlichen Bereichen) zur Einführung in die Lesson Study-Methode und/oder zur Kollaboration an der Entwicklung der Forschungsstunden.

Bereitstellen von Stunden für Planung und Durchführung des Unterrichts sowie für Interviews mit Schüler*innen und für Diskussion bzw. Weiterentwicklung der Forschungsstunden.

* Moderieren:

Auch wenn der Lesson Study-Zyklus eine klare Strukturierung aufweist, bedarf es bei der Planung einer genauen, detaillierten Klärung und Vereinbarung. Übernehmen der Moderation und Festschreiben der Ziele und Aufgaben.

* Präsentieren und Protokollieren:

Veröffentlichen bzw. Vorstellen der durchgeführten Lesson Study: Präsentieren der Ergebnisse und Protokolle im Rahmen einer Konferenz und/oder durch Verfasssen von Protokollen oder anderen schriftlichen Informationen durch das gesamte Team.

\section{Lesson Study - ein Erfahrungsbericht der Praxisvolksschule der PH NO̊}

In der Praxisvolksschule werden seit mehreren Jahren im Rahmen der Pädagogisch-Praktischen-Studien der Lehrer*innen-Ausbildung Lesson Studies durchgeführt. Auch in der Fort- 
und Weiterbildung finden fallweise Lesson Studies in der PVS statt. Dabei arbeiten die Klassenlehrer*innen mit den Kolleg*innen der Pädagogischen Hochschule zusammen.

Im Zuge dieser Praxiseinheiten und im Austausch mit Wissenspartnern konnten die Pädago$\mathrm{g}^{*}$ innen der PVS Kenntnisse über das Lesson Study-Konzept erwerben und Erfahrungen in der Durchführung sammeln.

Im Unterricht liegt der Fokus der PVS zunehmend auf dem Lernprozess der Schüler*innen mit dem Schwerpunkt, einen schüleraktiven und kompetenzorientierten Unterricht sowie leistungsdifferenziertes Arbeiten umzusetzen. Die steigenden Anforderungen an die Lehrpersonen und der Anspruch, das Lernangebot für die Schüler*innen im Sinne einer kontinuierlichen Unterrichtsentwicklung zu verbessern, führten dazu, auch innerhalb des PVS-Teams Lesson Study-Zyklen als Methode zur persönlichen Professionalisierung durchzuführen.

Nachdem diese Entscheidung getroffen wurde, war es nun die Aufgabe der Schulleiterin, die erforderlichen Rahmenbedingungen zu schaffen und Ressourcen zur Verfügung zu stellen, um die Durchführung zu ermöglichen und die Lehrer*innen dabei zu unterstützen. Es zeigte sich, dass die Schulleitung vor allem in der Vorbereitung und Planung eine wichtige Rolle spielt, indem sie unter anderem die Moderation übernimmt. Während sich die beteiligten Lehrpersonen vorrangig auf thematische und methodische Fragen konzentrieren, kann sie darüber hinaus inhaltliche, personelle und organisatorische Details ansprechen und zu deren Klärung beitragen. Hier sind Fragen zur Rollen- und Aufgabenverteilung sowie zur zeitlichen und räumlichen Einteilung gemeint. Innerhalb einer kleinen Schule stellen gerade diese Bereiche eine große Herausforderung dar und sind nur durch besonderes Engagement der Pädagog*innen zu meistern.

Alle Arbeiten rund um die Unterrichtsdurchführung und die kollegiale Hospitation - Besprechungen und Planungen, das Erstellen von Beobachtungsbögen und das Verfassen von Berichten - führten die Lehrer*innen neben ihren sonstigen pädagogischen und administrativen Aufgaben durch. Die beobachtende Teilnahme am Unterricht sowie die Interviews mit Kindern konnten während der Unterrichtszeit am Vormittag oder Nachmittag stattfinden, indem Teamlehrer*innen den Klassenunterricht übernahmen und der reguläre Stundenplan vorübergehend ausgesetzt wurde.

Die Schulleiterin begleitete die Kolleg*innen während des gesamten Prozesses, indem regelmäßig Erfahrungen ausgetauscht und kurzfristig notwendige Änderungen in der Organisation durchgeführt wurden. Dadurch konnte sie am „Lernprozess“ und Erfahrungszuwachs der Pädagog*innen teilhaben.

Die gewonnenen Erkenntnisse und die kollegiale Zusammenarbeit wurden als Bereicherung und wichtige Erfahrung erlebt. Die Sinnhaftigkeit und Möglichkeit der Unterrichtsentwicklung sind unbestritten. Allerdings bedarf es zusätzlicher zeitlicher Ressourcen für eine regelmäßige und umfassende Durchführung der Lesson Study-Zyklen und für die Zusammenarbeit von kollegialen Lerngruppen. Es wird als Auftrag an die Schulleitung gesehen, auf die Notwendigkeit dieser Unterstützung hinzuweisen und, soweit es möglich ist, die erforderliche Hilfe zur 
Verfügung zu stellen und die Lehrpersonen in organisatorischer, zeitlicher und überhaupt jeder Hinsicht zu unterstützen. Dadurch entsteht eine produktive und nachhaltige Zusammenarbeit zwischen Schulleitung und Kollegium.

\section{Erfahrungsbericht der Volksschule St. Johann im Lavanttal (Kärnten)}

Das Team der VS St. Johann setzte sich gemeinsam mit der Schulleitung zum Ziel, mithilfe von Lesson Study den Englischunterricht in der Grundstufe II durch den Einsatz der CLIL-Methode zu professionalisieren. Das Unterrichtskonzept von CLIL (Content and Language Integrated Learning) war den Pädagog*innen bereits bekannt. Neu war für die Pädagog*innen - wie für die Schulleitung - die Lesson Study. Indem Lesson Study das Augenmerk auf das Lernen legt, wird sie zur Forschung für das Lernen und gleichzeitig zum natürlichen Bestandteil einer förderlichen Lernumgebung (vgl. Mewald \& Rauscher, 2019).

Das Konzept von Lesson Study wurde den Pädagog*innen der Volksschule St. Johann im Rahmen einer pädagogischen Konferenz im Schuljahr 2018/19 präsentiert.

Die Durchführung der Lesson Study orientierte sich anhand folgender Stufen:

$>$ Vereinbarung von gemeinsamen Zielen in der Gruppe

$>$ Gemeinsame Planung einer Lesson Study-Forschungsstunde

$>$ Die Stunde abhalten, beobachten und gemeinsam nachbesprechen

$>$ Gemeinsame Überarbeitung der Lesson Study-Forschungsstunde

Reflexion und Austausch der Ergebnisse (Stepanek et al., 2006)

Die Abhaltung der Lesson Study zeigte der Schulleitung, welcher Mehrwert des Unterrichtens erzielt werden kann, wenn Pädagog*innen die Möglichkeit bekommen, sich in einer professionellen Lerngemeinschaft (PLG) gezielt mit einer Unterrichtsmethode auseinandersetzen zu können. Durch die gemeinsame Planung, Durchführung, Analyse und Auswertung der Ergebnisse wurde verdeutlicht, dass auch jede noch so gut geplante Stunde verbessert werden kann. Lesson Study ermöglichte Lehrenden einen Perspektivenwechsel, hin zum*zur Schüler*in. Manifestierte Meinungen und Vorurteile der Lehrer*innen wurden teilweise widerlegt und es wurde ihnen ermöglicht, den eigenen Unterricht zu öffnen und mit vielen Augen zu sehen, da nicht nur eine Person für die Durchführung und Beobachtung verantwortlich war.

Die Schulleitung bekam als Teil des professionellen Teams die Möglichkeit, das Unterrichtshandeln aus verschiedenen Perspektiven wahrzunehmen und auf Augenhöhe mit den Kolle$\mathrm{g}^{*}$ innen bestehende Unterrichtskonzepte zu überarbeiten oder neue Konzepte und Ideen zu erarbeiten sowie Problemfelder zu thematisieren. 
Indem jede PLG an einem Thema forschte, entstand ein Verständnis für die Unterrichtsentwicklung am Schulstandort. Im Bereich der Durchführung war die Schulleitung als Teil des gesamten Teams zu sehen. Die Schulleiterin gab die Rolle des Coaches ab und vermittelte den Pädagog*innen dadurch das Gefühl, dass jede*r Einzelne ein gleichwertiger Teil der Gruppe war. Es sollte ein freier unkonventioneller Austausch der Ideen möglich sein, ohne dass geurteilt bzw. beurteilt wurde. Dadurch konnten Ideen eingebracht und die Zusammenarbeit des gesamten Teams in Form einer professionelle Lerngemeinschaft nachhaltig gefördert werden.

Die Schaffung der Rahmenbedingungen oblag der Schulleitung. Es war erforderlich, das gesamte Team zu schulen, sodass das Kollegium selbst als professionelles Team diese Form der Unterrichtsentwicklung in Zukunft durchführen kann. Lesson Study wurde an der VS St. Johann auch in den Entwicklungsplan aufgenommen, damit eine längerfristige Zielsetzung ermöglicht wird.

Jährlich sind pro Grundstufe zwei Lesson Study-Zyklen vorgesehen. Die Schulleitung unterstützt das Team auf dem Weg zur Professionalisierung.

Der Schulleitung ist es ein Anliegen, Lesson Study auszudehnen und eine Zusammenarbeit mit weiteren Schulen anzustreben, damit ein schulübergreifender Erfahrungsaustausch angebahnt wird. Neue Ideen und Sichtweisen von Kolleg*innen aus anderen Schulen könnten in das Unterrichtshandeln der Pädagog*innen am jeweiligen Schulstandort aufgenommen werden. In den vergangenen Jahren wurden bereits schulübergreifende Lesson Studies durchgeführt und die Pädagog*innen waren bereit, auch in ihrer Freizeit daran teilzunehmen.

Des Weiteren wäre es für die Schulleitung eine Bereicherung, Expert*innen aus der Politik oder der Schulaufsicht einzuladen, sodass diese die Möglichkeit bekommen würden, die Forschungsstunden zu beobachten und dadurch sofortiges Feedback darüber zu erhalten, wie Schüler*innen und Lehrer*innen mit neuen Themen und Ansätzen arbeiten oder auch unter anderem mit neuen Bildungszielen Schwierigkeiten haben. Darüber hinaus würden politische Entscheidungsträger, Lehrer*innen und Forscher*innen ein gemeinsames Verständnis der Standards, basierend auf Erfahrungen, die sie alle gesehen und diskutiert haben, entwickeln.

\section{Resümee}

Für Elliott (2012) ist der Ausgangspunkt einer Lesson Study das Bedürfnis, eine Unterrichtssituation, die subjektiv nicht optimal erlebt wird, durch eine kollegiale Zusammenarbeit zu professionalisieren.

Anhand einer gemeinsamen Planung und einer gemeinsamen Evaluierung der Unterrichtseinheiten ist für die Pädagog*innen eine Mitbestimmung im Schulentwicklungsprozess gegeben. Der fachliche Austausch innerhalb der Gruppe fördert das Unterrichtshandeln der Pädagog*innen. 
Lesson Study bietet gemäß Kiper (2012) insofern die Möglichkeit, individuelle Probleme an den Schulstandorten zu erörtern sowie neue Lernstrategien und Methoden zu etablieren. Es dient der Lernförderlichkeit, die eine Klärung und Anpassung am Schulstandort erfordert, und wirkt einer generellen und abstrakten Top-down-Regelung entgegen. Als Ausgangspunkt der Gestaltungsmaßnahmen wirkt der Bottom-up-Ansatz. Durch Lesson Study kann in der Unterrichtsentwicklung an den Schulen ein dynamischer Prozess, von der Basis beginnend, aufgebaut werden. Durch die Betrachtung der Unterrichtsentwicklung aus verschiedenen Blickwinkeln, von möglichst allen Beteiligten, eröffnen sich vielfältige Handlungsansätze. Anhand des Erfahrungsaustausches und der gegenseitigen Unterstützung der Pädagog*innen entsteht eine Dynamik mit neuen Entwicklungsmöglichkeiten. Die Unterrichtsentwicklung, die laut Clivaz und Takahashi (2018) einer gewissen Trägheit unterliegt, zeigt durch den Einsatz von Lesson Study, dass Pädagog*innen motiviert sind, pädagogische Ansätze nachhaltig zu integrieren.

Ein regelmäßiger Einsatz von Lesson Study ermöglicht eine positive Unterrichts- und Schulentwicklung. Letztlich muss jedoch betont werden, dass für die Umsetzung und die konsequente Durchführung die Schulleitung durch das Schaffen der Rahmenbedingungen verantwortlich ist.

\section{Literaturverzeichnis}

Altrichter, H. \& Posch, P. (2007). Lehrerinnen und Lehrer erforschen ihren Unterricht. Regensburg: Julius Klinkhardt.

Clivaz, S. \& Takahashi, A. (2018). Mathematics Lesson Study Around the World: Conclusions and Looking Ahead. In: M. Quaresma, C. Winsløw, S. Clivaz, J.P. da Ponte, N. Shúilleabháin \& A. Takahashi (Hrsg.), Mathematics lesson study around the world: Theoretical and methodological issues (pp. 153164). Cham.

Elliott, J. (2012). Developing a science of teaching through lesson study. International Journal for Lesson and Learning Studies, 1(2), 108-125.

Helmke, A (2015). Unterrichtsqualität und Lehrerprofessionalität. Diagnose, Evaluation und Verbesserung des Unterrichts. 6. überarbeitete Auflage. Seelze: Klett-Kallmeyer.

Keow Ngang, T. \& Chap Sama, L. (2015). Principal Support in Lesson Study. Procedia-Social and Behavioral Sciences 205, 134-139.

Kiper, H. (2012). Unterrichtsentwicklung. (Praxiswissen Bildung) Stuttgart: Kohlhammer.

Mewald, C. \& Rauscher, E. (Hrsg.) (2019). Lesson Study. Das Handbuch für kollaborative Unterrichtsentwicklung und Lernforschung. Innsbruck: Studienverlag. (Pädagogik für Niederösterreich 8)

Stepanek, J., Appel, G., Leong, M., Mitchel, M. \& Turner, M. (2006). Leading Lesson Study: A Practical Guide for Teachers and Facilities. Thousand Oaks: Corwin Press.

Schulunterrichtsgesetz: § 56 SchUG Schulleitung, Schulcluster-Leitung (Stand 15.8.2021) 


\section{Autorinnen}

\section{Silvia Quendler, MA MSc}

ist prov. Schulleiterin einer sechsklassigen Volksschule in Kärnten. Für die Bildungsdirektion Kärnten arbeitete sie als Mentorin für den Grundkompetenzaufbau Englisch (GK 2/4). Sie ist als Referentin in den Bereichen „Sprachbewusster Unterricht“ und „Englisch in der Grundschule" an der PH Kärnten und der PH Steiermark tätig.

Kontakt: silviaquendler@gmail.com

Angelika Dobrowsky, Mag.

Leiterin der Praxisvolksschule der Pädagogischen Hochschule Niederösterreich in Baden. Kontakt: angelika.dobrowsky@ph-noe.ac.at 